\title{
Spectral Evolution of Long-Period Fiber Grating During Written Process and Their Influence in the Sensitivity
}

\author{
A. Quintela*, M. A. Quintela*, D. A. Gonzalez*,S. Diaz **, M. López-Amo **, J.M. López-Higuera* \\ *Photonic Engineering Group-University of Cantabria \\ Avda. Los Castros s/ $n$ - C.P. 39005 Santander, Spain \\ Tel: ++ 34-42-201495; Fax: ++34-42-200877 Email: quintela@ unican.es \\ **Dept. of IEE-Public University of Navarra \\ Campus de Arrosadía, s/n- C.P.31006 Pamplona, Spain
}

\begin{abstract}
The peak loss wavelength shift, attenuation and bandwidth values on a LPG during the UV multi-pass writing process can be used to estimate the LPG sensitivities in real-time during the fabrication task.

OCIS codes:(060.2370) Fiber Optics Sensor; (050.2770) Gratings.
\end{abstract}

\section{INTRODUCTION}

The long period gratings (LPG) can be understood as a perturbation of the core refractive index of the optical fiber with a modulation period much longer than the light wavelength. This period can be found, typically in the range from $100 \mu \mathrm{m}$ to $1 \mathrm{~mm}$. The period of the index modulation is such that it satisfies a phase matching condition between the fundamental core mo de and a co-propagating cladding mode for specific wavelengths. The LPG's have been used in multiple optical applications, for example, as band rejection filters [1], gain equalizers [2] and sensors for physical parameters such as temperature, strain, refractive index and curvature [3].

The UV-induced index modulation is the most widely used method for the fabrication of the LPGs. The refractive index modulation may be built up using several procedures. The amplitude-mask writing technique is the most widely used due to its reliability [1]. The disadvantage of this technique is that the change of the LPG period could be realized only by using different periodicities of amplitude-mask. The other fabrication technique is the point-by-point UV-writing process [4]. In this process, the periodic exposure is carried out by a computer-controlled shutter program. An advantage of this technique is the great flexibility because a LPG of arbitrary periodicity and length can readily be written by appropriately programming the shutter and translation stage. The main disadvantage of using the point-by-point writing method is that the technical requirements are strict.

Currently, using simulation programs the LPG behaviors can be theoretically estimated [5]. The resonant wavelengths and the sensitivity factor are can be approached as a function of the LPG period. In order to be used the LPG structure as a transducer fiber element for sensors this procedure permits to obtain approximately the LPG behavior in the presence of the external parameters and, therefore select the design conditions as a function of the established requests. From here on, the fabrication of the specific LPG and its sensitivity characterization to an external para meter can be estimated. However, the real values of these mentioned parameters can be different that the theoretical calculated. So it is necessary to have a method than permits to check, in real-time, the mentioned LPG sensitivities during the fabrication process.

The evolution of the spectral transmission behaviors and, in particular the resonant wavelengths, when the induced core index changes, provides reliable information about external parameters response of LPGs. In this paper the experimental resonant wavelength shifts as a function of grating refractive index increases during a multipass fabrication are reported. From this experimental information, the sensitivities of the LPG resonant wavelengths can be measured on-line after each UV- writing pass of the proposed fabrication process.

\section{THEORY}

The resonant wavelengths $\left(\lambda_{\text {res }}\right)$ of a LPG with period $\Lambda$ are given by:

$$
\lambda_{\text {res }}^{i}=\left(n_{\text {eff }}(\lambda)-n_{\text {cladd }}^{i}(\lambda)\right) \Lambda
$$


where $n_{\text {eff }}$ is the effective index of the fundamental core mode, $n_{\text {cladd }}^{i}$ is the effective index of the $\mathrm{i}^{\text {th }}$-order cladding mode.

The $\lambda$ dependence of the effective indices is due to material and waveguide dispersion being the later the dominant dispersion component, in this work. The difference between core effective index dispersion and the cladding effective index dispersion is the most important parameter of this study. It can be assumed that the material dispersion have the same effect on $n_{\text {core }}$ as well as $n_{\text {cladd.. }}$ So when the difference between the refractive indices is taken, the waveguide dispersion is the dominant factor to LPG spectra evolution

During the LPG fabrication, the wavelength $\left(\lambda_{\text {res-max }}\right)$ at which resonant coupling occurs when the UV induced core refractive index increase can be defined as [4]

$$
\lambda_{\text {res-max }}^{i}=\left(n_{\text {core }}\left(\lambda_{\text {res-max }}\right)+\delta n_{\text {core }}-n_{\text {cladd }}\left(\lambda_{\text {res-max }}\right)-\delta n_{\text {cladd }}\right) \Lambda
$$

$\delta n_{\text {core }}$ is the average index modulation over the length of the LPG and $\delta n_{\text {cladd }}$ is the change in the effective index of the cladding mode as a result of the core refractive index change. This term is very close to zero and can be negligible.

If the core and cladding effective indices are expanded in a Taylor series about $\lambda_{\text {res }}$, the expression (2) can be rewritten as [6]

$$
\begin{gathered}
\lambda_{\text {res }-\max }^{i}=\lambda_{\text {res }}^{i}\left(1+\frac{\delta n_{\text {core }}}{n_{\text {core }}-n_{\text {cladd }}^{i}} \cdot \frac{\frac{d \lambda_{\text {res }}}{d \Lambda}}{n_{\text {core }}-n_{\text {cladd }}^{i}}\right)=\lambda_{\text {res }}\left(1+\frac{\delta n_{\text {core }}}{n_{\text {core }}-n_{\text {cladd }}^{i}} \cdot \gamma^{i}\right) \\
\gamma^{i}=\frac{\frac{d \lambda_{\text {res }}}{d \Lambda}}{n_{\text {core }}-n_{\text {cladd }}^{i}}
\end{gathered}
$$

The factor $\gamma$ represents the contribution of the waveguide dispersion of the $\dot{i}_{\text {h }}$ core-cladding. On the other hand, this one has determinant influence on the LPG sensor design. It is related directly with the LPG sensitivity factor $\left(S_{p}\right)$ through the expression [5]:

$$
S_{p}=d \lambda_{r e s} / d p=\lambda_{r e s} \cdot \gamma \cdot K_{p}
$$

where $p$ is the specific external perturbation (temperature, strain, bend, etc) and $K$ is a constant depending on the perturbation.

The evolutions of the $\lambda_{\text {res-max }}$ during the LPG multi-pass fabrication process will provide very important information about the factor $\gamma$, for every cladding mode, as a consequence, the LPG level of response to external perturbations can be obtained.

\section{EXPERIMENTAL}

In order to study the LPG transmission spectrum evolution during the writing process is used a multi-pass fabrication technique. The method used is point-to-point, based in the synchronization of a shutter with a continuous scan movement of the UV beam along the fiber. The photosensitive fiber used was an hydrogenate standard telecommunications fiber. A continuous wavelength argon ion laser at $244 \mathrm{~nm}$ is used. All LPG's were written with the same UV pass scan speed, and with a duty cycle equal to $50 \%$. The LPG used periods were in the range between $140 \mu \mathrm{m}$ to $450 \mu \mathrm{m}$. The number of pass during the fabrication process oscillated between 4 and 12, in function of period.

For each cladding mode coupling there are two loss peaks in the LPG transmission spectrum. With an increase in the LPG period the separation between the twoloss peaks decrease, just to arrive to a period where 
both peak are in the same position on the spectrum In this moment an increase on the period cause the disappearance of this loss peak, and this cladding mode is not coupled.

During the LPG fabrication process it is observed that the wavelength shift of the LPG loss peak between two consecutive pass was bigger as smallest was the distance between the two loss peaks for a cladding mode. The evolution of transmission spectrum of a sample of four LPGcan be observed on figure 1. In the graph (a) -period equal to $161 \mu \mathrm{m}$ - can be observed how for the mode coupling $\left(\mathrm{LP}_{0,12}\right)$ between the third an the ninth pass the two loss peaks of coupling mode are successively approaching and, just in the tenth pass, the two loss peaks are joined at $1390 \mathrm{~nm}$. With neither more UV writing passes this mode is nor coupling anymore. The loss peak bandwidth and the wavelength shift between two consecutive pass increases with the number of pass too. In the second graph (b) the period is $173 \mu \mathrm{m}$, and it can be observed as the bigger attenuation is for the mode $\mathrm{LP}_{0,11}$, but only is seen one of the loss peak, because the other is a wavelength bigger $\tan 1700 \mathrm{~nm}$. Though with a period of $185 \mu \mathrm{m}$, graph (c), the behavior of the mode $\mathrm{LP}_{0,11}$ is similar as the behavior of mode $\mathrm{LP}_{0,12}$ with a period of $161 \mu \mathrm{m}$, but in a gre ater wavelength. Finally in the graph (d), the period of LPG is 210 $\mu \mathrm{m}$, can be observed the evolution of the loss peaks for the modes $\mathrm{LP}_{0,10}, \mathrm{LP}_{0,9}$ and $\mathrm{LP}_{0,8}$. Another important conclusion obtained from this four graph is as bigger as the mode is necessary more number of passes in order to obtain the same attenuation of the loss peak in the transmission spectrum

From the spectrums of transmission obtained is possible to obtain for each pass the curve of the dependence between the wavelength loss peaks and the period of a LPG. In the Fig. 2 (a) it is represented this dependence for the third pass.
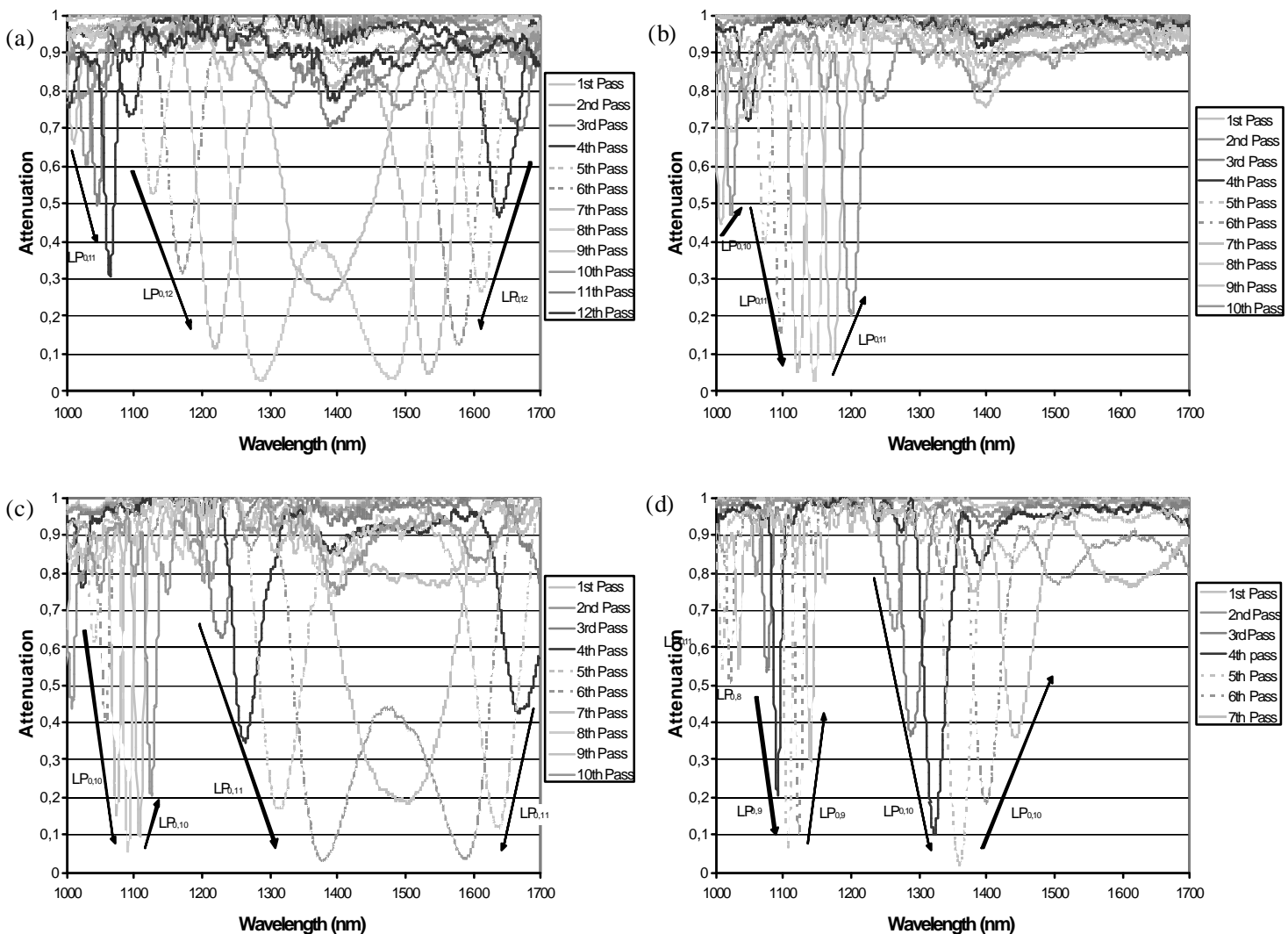

Fig. 1 Evolutions of transmissions spectrums of four LPG during multipass fabrication process. (a) LPG period equal to $161 \mu \mathrm{m}$ (b) LPG period equal to $173 \mu \mathrm{m}$ (c) LPG period equal to $185 \mu \mathrm{m}$ (d) LPG period equal to $210 \mu \mathrm{m}$ 
We can observe how effectively, as indicated the equation (3), the wavelength shift increases in accordance with the parameter $\gamma$, as well as with the slope in Fig. 2 (a). On the other hand, for a cladding mode coupling, as increase the number of pass the distance between the two loss peaks decrease as well, and so increase the value of parameter $\gamma$. That can be shown in Fig. $2(\mathrm{~b})$, where it is represented for $3^{\text {rd }}$ and $6^{\text {th }}$ pass the dependence of resonant wavelength with the LPG period
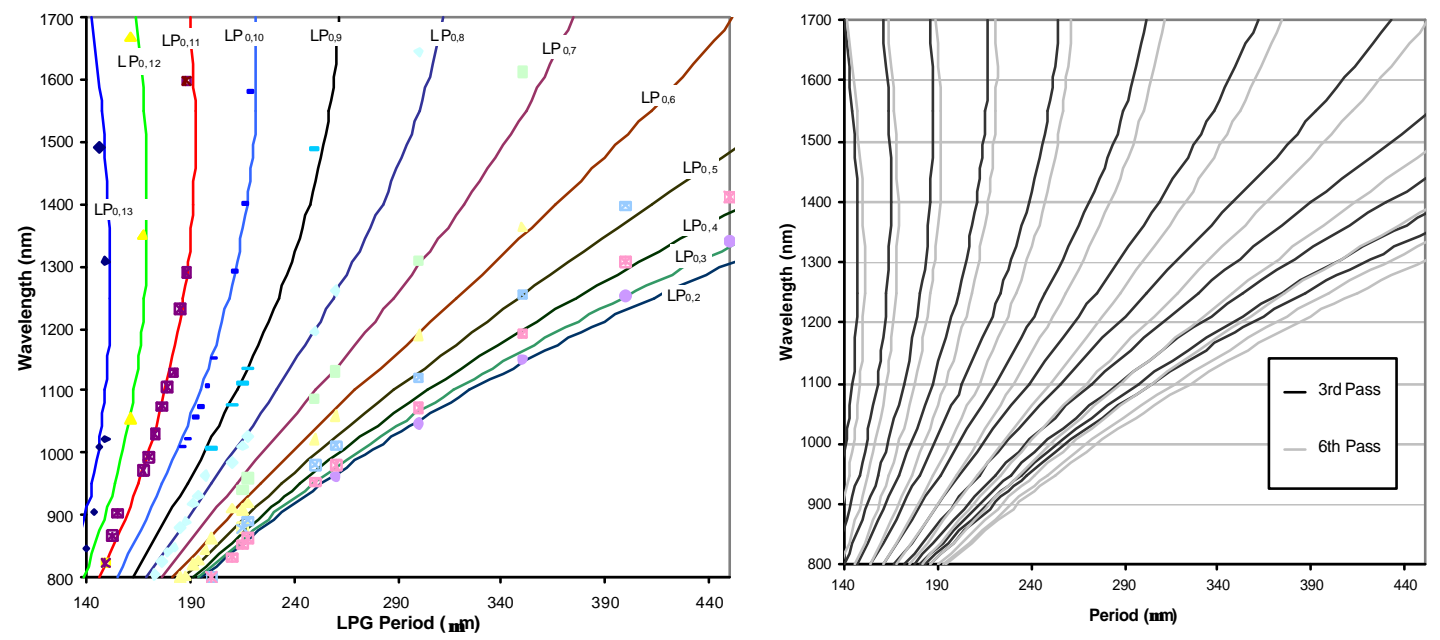

Fig. 2 (a) Experimental(continuous line) and simulated (discrete points) dependence of the mode resonance wavelengths with the LPG periods for a given UV-writing pass. (b) Evolution of the resonance wavelengths for $3^{\text {rd }}$ and $6^{\text {th }}$ pass

So as the LPG sensitivities depends directly of the parameter $\gamma$, equation (4), it can be measure the wavelength shift between two consecutive pass, controlling in realtime the sensitivity of the LPG written , and to realize the required number of pass necessary in order to have the desired sensitivity.

\section{CONCLUSIONS}

The evolutions of the peaks loss wavelength shift, attenuations and bandwidths in function of the increase of the modulation core index of a LPG period have been experimentally obtained. For each cladding mode there are two loss peaks in the LPG transmission spectrum. Writing the LPG by means a multi-pass fabrication technique the wavelength shift and the bandwidth of the two peaks loss for each coupling cladding mode are inversely proportional to the distance between them. Furthermore, as the different LPG sensitivities are proportional to this wavelength shift, an online control of the sensitivities of a LPG, after each pass of the multi-pass writing fabrication process, can be done.

\section{ACKNOWLEDGEMENTS}

This work has been co-supported by the Spanish TEC2004-05936-C02 and TEC2005-08218-C02-02 projects.

\section{REFERENCES}

[1] A. M. Vengsarkar, P. J. Lemaire, J. B. Judkins, V. Bhatia, T. Erdogan and J. E. Sipe, "Long period fiber gratings as band rejection filters", J. Lightwave Technol., Vol. 14, 58-64, 1996.

[2] Kashyap, Fiber Bragg Gratings, Harcourt Brace \& Company, New York, 1999.

[3] V. Bhatia, "Applications of long-period gratings to single and multiparameter sensing", Optics Express, Vol. 4, No. 11, 457-466, 1999.

[4] L. Zhang,Y. Liu, L. Everall, J. A. R. Williams and I. Bennion, "Design and realization of long-period grating devices in conventional and high birefringence fibre and their novel applications as fibre-optic load sensor". IEEE J. Selected Topics in Quantumm Electron, Vol.5, 1373-1378, 1999.

[5] X. Shu, L. Zhang and I. Benn, "Sensitivity characteristics of longPeriod fiber grating", Journal of Lightwave Technology, Vol. 20, No. 2, 255-266, 2002.

[6] T. W. Macdougall, S. Pilevar, C. W. Haggans and M. A. Jackson, "Generalized expression for the growth of long period gratings", IEEE Photonics Technology Letters, Vol. 10, No. 10, 1449-1451, 1998 